\title{
Criterion-Referenced Value-Added Scores
}

\author{
Mark C. White ${ }^{1}$ \\ Emanuele Bardelli ${ }^{1}$
}

${ }^{1}$ University of Michigan

\begin{abstract}
Author note
Correspondence concerning this article should be addressed to Mark C. White, University of Oslo, Postboks 1099, Blindern 0317, OSLO. E-mail:

mark.white@ils.uio.no. Phone: +47-22844498.
\end{abstract}




\begin{abstract}
Value-added (VA) scores signal teacher quality and are designed to be used, with other data, to make employment decisions. However, this usefulness is hampered by: 1) their normative nature, which promotes competition among teachers, 2) their difficulty to interpret and incorporate with other data, which makes using VA scores as one data source among many difficult, and 3) a lack of trust in scores. We show how to create (model-based) non-normative and more interpretable VA scores, which winds up being a simple rescaling of traditional VA scores. Our approach more closely links VA scores to data-based decision making efforts and enables the creation of clear and objective criterion of effectiveness for teachers.

Keywords: value-added, criterion-referenced, data-based decision making, benchmark assessment
\end{abstract}


Much has been written about value-added (VA) scores, both by advocates who promote the usefulness of VA scores and by critics who worry about the negative impacts VA scores may have on education when used in a framework of high-stakes accountability. One contentious topic in this debate, which we take up in this paper, is that "almost none of the technical issues that currently occupy the attention of researchers are likely to have much impact on how VAM results are received and interpreted by teachers and principals" (Braun, 2015). This is especially troubling as the way VA scores are interpreted has a large impact on the effectiveness of any teacher evaluation system (Goldhaber, 2015).

There are three areas of concern about VA scores that we take up here. First, VA scores are presented on a normative scale that compares teachers to other teachers, which may hinder cooperation between teachers (e.g. Cohen \& Goldhaber, 2016; Collins, 2014; Kappler Hewitt, 2015; Johnson, 2015). Second, practitioners have trouble making sense of VA scores as these scores are usually reported on scales that have little connection to practical measures of teaching (e.g. Braun, 2015; Cohen \& Goldhaber, 2016; Collins, 2014; Goldring, et al., 2015). Third, there is a lack of trust in VA scores, due to fluctuations in scores across time, the complexity and lack of transparency of scores, and other factors (e.g. Amrein-Beardsley \& Collins, 2012; Collins, 2014). Of course, these three concerns are not the only issues affecting VA score interpretability and usefulness (see, e.g., Braun, 2015; Johnson, 2015). Many conceptual and technical concerns exist in VA scores. To appropriately scope this paper, however, we focus only on these three challenges concerns.

In this conceptual paper, we start from the way VA scores should be used from a signaling perspective and build a new way to generate scores that addresses these three concerns. We will argue that the signaling power of VA scores will be increased when the scores are embedded within a larger system that connects differences in scores to 
instructional implications. Connecting VA scores to data use practices already in place in most schools, such as benchmark assessment systems and data-based decision-making, requires a new way of estimating VA scores. Importantly, this new approach winds up being a simple rescaling of VA scores, which is ideal in some sense as current statistical models and known properties of current VA score are retained (including the many known issues with VA scores). The resulting scores are not only arguably more interpretable, but are both model-based estimates of non-normative scores, such that one teacher's scores are arguably independent of other teachers, and gain a criterion-referenced interpretation, allowing the identification of teachers whose students show a year of growth on test scores. As such, we term these new VA scores criterion-referenced VA scores (CR-VA). We turn now to describing the intended uses of VA scores, highlighting the conceptual foundation of our proposed approach. We then describe the method of creating the new type of VA scores. We conclude with a discussion of the possible implications of the new scores.

\section{Intended Use of Value-Added (VA) Scores}

VA scores are meant to provide information on teacher quality that practitioners can use, as one data source among many, to determine if teachers are effective (at promoting high test scores $)^{1}$. To be an effective signal, VA scores must be useful both to administrators making employment decisions and to teachers as an indicator of their instructional quality. Arguably, VA scores, as currently constructed, are not well suited to this task (Goldhaber, 2015). Leaving aside concerns about reliability and potential bias in VA scores for the moment, the fact that VA scores are often presented on a standard normal scale (i.e. mean 0 and standard deviation 1) makes it difficult for decision-makers to fully make sense of data and to combine information from VA scores with other information (Goldring, et al., 2015). Let's assume a principal is deciding between hiring two teachers, one with a VA of 0.2 and one with a VA of 0.3 . Clearly the second teacher's students demonstrate greater growth on 
test scores, but how much of a difference is this in terms of student learning? Does the second teacher's VA score override the fact that the first teacher has more experience with students similar to those in the school, has a broader certification, or coaches football? How much of a difference in VA scores would be necessary to overcome these differences? Without some basis for understanding the implication of a 0.1 difference in VA scores in terms of student achievement, decision-makers will have trouble appropriately using VA scores in conjunction with other data to address the schools' multiple needs and goals. Further, while it is clear that both of these teachers are above average at raising student test scores, it is not clear (from the VA scores) if either is an objectively effective teacher.

A basic condition for VA scores to be a maximally useful signal, then, is that individual scores and differences between scores are interpretable. This requires presenting VA scores on a carefully considered scale that is familiar to practitioners. The typical standard normal scale has some benefits (e.g. a clear definition of the average teacher; a clear measure of the scale's standard deviation), but differences between scores are difficult to interpret, all interpretations emphasize the normative nature of scores, and practitioners have little experience with standardized scores. Ways of addressing these shortcomings have been attempted, typically by trying to leverage the student scale score metric, which must be on a proficiency scale with four categories of proficiency. For example, New York used a 1-4 proficiency scale, reporting both expected and observed average scores for a teacher, framing VA scores as the difference between students' average expected and observed scores (Corcoran, 2010). Given the 1-4 scale, a teacher with a VA score of 0.2 has students who scored $1 / 5$ of the gap between proficient and advanced performance higher than expected (note that this particular scaling makes the assumption that gaps between all proficiency levels are equal). 
While this scaling could add interpretability by anchoring scores as differences between two clearly defined proficiency thresholds, it is not clear practitioners have the knowledge or experience needed to make sense of this scale. Further, while the way scores are presented de-emphasizes the normative nature of the VA score (by focusing attention on the proficiency scale), the scores are still purely normative. This can be seen by holding the scores of one teacher's students constant (and hence teacher quality as measured by VA scores) and increasing test scores of students for all other teachers, the predicted test scores for the focal teacher will increase (due to population level growth in scores) while the observed scores remain constant, and so the teacher's VA score will decrease (despite us holding student learning [i.e. "teacher quality"] constant). Thus, the teacher's "value-added" changed when their quality did not, a characteristic of normative scales.

This scaling approach does, though, suggest a potential solution to the challenge of interpreting VA scores. If VA scores could be projected onto a scale such that practitioners could understand the student learning and instructional implications of score values, VA scores would become far more interpretable. One useful scale is the scale score upon which student standardized tests scores are placed. This is because teachers, on a yearly basis, observe the scores that their students, who they know well, receive. This lets teachers develop some basic notion connecting specific score values to students' knowledge. In the next section, we argue that data-based decision-making and benchmark assessments can be leveraged to further develop teachers' deep understanding of the student scale score metric.

\section{Data Use and Benchmark Assessments}

There is a growing push for greater data use in schools (Beaver \& Weinbaum, 2015). Specifically, benchmark assessments are being used by many schools to track students' learning progress. The outputs of benchmark assessments come in many forms. Often, they present progress students make towards specific standards (e.g. percent proficient for specific 
standards) with cut-scores indicating expected scores at various points during the year. However, in rare cases, upon which we focus here, benchmark assessments present predicted scores on the end of year accountability test on the student scale score metric. This is especially likely when the benchmark assessments are provided by the end of year test providers (e.g. SMARTER BALANCED, and PARCC), though some private benchmark assessment providers also report scores in this way (see https://www.te21.com/samples for examples from one provider across many states). This (to our knowledge uncommon) practice can be leveraged to help build practitioners' understanding of the learning and instructional implications of different scores on the student scale score metric.

Schools generally administer benchmark assessments multiple times throughout the year, using them to select students into various intervention efforts (Beaver \& Weinbaum, 2015; Booher-Jennings, 2005; Farrell \& Marsh, 2016; Wachen, Harrison, \& Cohen-Vogel, 2018). In doing so, practitioners observe how scores change as the result of various instructional interventions. If benchmark assessments were placed on the student scale score metric, seeing how interventions change predicted scores would further develop practitioners' ability to reason about this metric, allowing them to connect various intervention efforts to differences in predicted scores. For example, teachers may observe that after-school tutoring tends to increase a students' predicted score by about 5 scale score points while assigning students to an extra intervention block tends to increase scores by about 10 scale score points. This sort of reasoning, which is growing more common, especially in schools using databased decision making, is the sort of experience necessary to interpret VA scores and differences in VA scores if the VA scores were placed on the same scale.

Of course, this sort of reasoning about scores and various interventions is only likely to strongly develop in school settings where effective data use practices have been adopted. However, given the push for data use in schools, the number of such settings should increase. 
In fact, the demands here on both the benchmark assessment and on practitioners' data use capabilities are quite modest. All we need here is for the benchmark assessment to reasonably accurately predict students' scores on the end of year exam and for these predictions to connect with interventions ${ }^{2}$. This is because we are focusing solely on the role benchmarks and data-based decisions can make on building teacher's understanding of the underlying student scale score metric so that VA scores become more interpretable rather that the broader role these practices should play in education, which is beyond the scope of this current paper.

\section{Creating a Value-Added Score Based on a Student's Predicted Score}

Up to this point, we have argued for placing VA scores on a scale that is familiar to practitioners to support their interpretation and understanding of scores. We have argued for using the student scale score metric as this scale since teachers already see the scores that students they know well receive on this scale. We have further argued that benchmark assessments and data-based decision-making initiatives can be leveraged to further build practitioners' familiarity with this scale. While this might build interpretability of VA scores if scores are placed on the student scale score metric, this does not solve issues about the normative nature of VA scores.

Fundamentally, VA scores are normative because teachers teach different sets of students in different contexts, requiring statistical models to adjust for differences in the students being taught across teachers. These models adjust for student factors affecting test scores, estimating average deviations caused by teachers from students' predicted achievement score. These deviations are considered necessarily normative because they are deviations from expected scores estimated by models using the full population of students (i.e. they are deviations from the normal progress). 
The key to VA scores that are not normative, then, involves crafting scores not based on the difference between observed and expected scores while retaining necessary statistical adjustments for the fact that teachers teach different students. We propose a solution here that uses the estimated score of a common hypothetical student that is taught by every teacher as the teacher's VA score. Consider the potential outcomes interpretation of VA scores (see Rubin, Stuart, \& Zanutto, 2004), which starts from the premise that each student has a potential achievement score that they would receive in each teacher's classroom but only one of these potential outcomes is observed. From this perspective, we might imagine that we could observe the average score that a class of students would receive were they taught by each teacher. The average score this class would receive could be used as the VA score for each teacher, in so far as this average score allows us to compare test score outcomes across teachers, attributing differences to teacher quality. Statistical adjustments in this scenario are unneeded since each teacher teaches the same class of students (we might also hold teaching context constant in this hypothetical). Further, under SUTVA, the average score would be non-normative (SUTVA is necessary for all VA scores, though not uncontested; Reardon \& Raudenbush, 2009).

In reality, we observe only a single outcome for each student and the VA statistical model must be relied upon to adjust for the potential outcomes that are unobservable. However, given a statistical model that adjusts for these differences (a necessary assumption for all VA scores), we could generate a model-based potential outcome estimate for a given student in each teachers' classroom. By selecting a hypothetical student and estimating their potential outcomes in each classroom, we get teacher specific scores that are adjusted for the fact that teachers teach different students. We propose these model-based potential outcome estimates as VA scores, calling the hypothetical student used to create scores the "standardizing student," which, it must be noted, is a hypothetical construct created by 
specifying student characteristics (see below) and not an actual person. Importantly, the potential outcomes of the standardizing student in each teacher's classroom (i.e. our proposed VA score estimate) both adjusts for the differences in the specific students each teacher teaches and is a model-based estimate of non-normative scores, meaning a teacher's score is independent of other teacher's scores (assuming of course the VA model is correct and SUTVA -- an assumption needed for all VA scores).

This makes the VA estimate based on the predicted score of the standardizing student non-normative, alleviating concerns about VA scores hampering teacher cooperation. Further, assuming the VA model uses student scale scores (this is not a necessary assumption, see below), the score will be on the student scale score metric naturally, which practitioners should have experience making sense of, especially if an aligned data-based decision-making initiative were also adopted (see the previous section). Thus, the first two concerns about VA scores discussed earlier, their normative nature and the trouble practitioners have making sense of scores, can be addressed under this approach. Enacting this approach, though, requires selecting a standardizing student whose potential test score in each teacher's classroom can be predicted, a problem we turn to after discussing the third concern about VA scores.

\section{Building Trust in Value-Added Scores}

This leaves only the third concern of VA scores raised earlier: teachers have little trust in scores (Amrein-Beardsley \& Collins, 2012; Collins, 2014; Wachen et al., 2018). A conceptual paper such as this one cannot demonstrate that this new approach to VA scores will or will not build trust in scores, but there is some reason to be optimistic. First, the approach described here places VA scores on a student scale score metric rather than the teacher standard deviation metric. Teachers account for roughly $1-14 \%$ of the variance in student test scores (American Statistical Association, 2014) so that a 1 standard deviation 
shift on the current VA score scale is only a $\sim 0.1-0.37$ standard deviation shift on the student scale score metric. Thus, large shifts in the teacher distribution correspond to relatively smaller shifts on the student scale score metric. This should make scores appear more stable across time (note that this change is based solely on the frame in which scores are presented and not the scores nature), which is a clear benefit since widely varying scores across years is a major source of distrust (Collins, 2014).

Second, the shift to viewing a VA score as a predicted score for a given student suggests a new way of presenting scores. Teachers can be shown the performance of their students alongside predicted performances and appropriate confidence intervals. As teachers see the models accurately predict their own students' performance, even those perceived as especially challenging whom teachers might have thought were the cause for lower scores this year, some confidence in the accuracy of scores might be generated. Similarly, teachers whose students' scores are not accurately predicted can legitimately raise concerns about their scores. Multiple iterations of this sort of score portrayal will be necessary to iron out details of presentation.

While there is some reason for optimism, any potential gains towards the problem of trust in VA scores from the changes introduced here will be quite modest because many other factors diminish trust in VA scores (e.g. Kappler Hewitt, 2015) and a school's overall relational trust is a major determinant of how teachers will react to scores (Bryk \& Schneider, 2002).

\section{Specifying a "Standardizing Student" to Generate Criterion-Referenced Scores}

There is a need to specify the standardizing student whose potential outcomes will be estimated for each teacher and used as their VA score. This amounts to, in effect, fixing the values of student covariates contained in the VA model. For the most commonly used VA models, this requires only fixing students' prior test scores (e.g. EVAAS and growth 
percentile models; Collins \& Amrein-Beardsley, 2014), but, for econometric models, this requires fixing student characteristics, such as race, free lunch status, etc. The choice of specific values is arbitrary, though careful selection can add to the meaning of scores.

Within the "one best system" (Tyack, 1974), schools are divided into specific grades with specific knowledge to be learned in each grade. This is even truer in current standardsbased accountability systems. Due to NCLB and now ESSA, all states have grade level standards with explicitly stated proficiency cut-scores. According to the logic of this system, one important aspect of a teacher's job is to take a student who has achieved proficiency in the previous grades and ensure they reach proficiency in the content taught in the current grade.

This suggests fixing the standardizing student's previous test scores to the proficiency cut-score for the previous grades. This "barely proficient" standardizing student should then be expected to receive a proficient score on the end of year test if taught in a classroom where students learn a year's worth of material from their teacher. That is, the current year's proficiency cut-point becomes a cut-score that can be used to determine whether a teacher's students are demonstrating a year's worth of test score growth. In classrooms where the barely proficient student is predicted to score at or above the proficiency cut-point, students have learned at least a year's worth of content. This factor is why we choose to call this method of creating VA scores criterion-referenced VA (CR-VA) scores. CR-VA scores have a clear, meaningful threshold based on the inherent logic of the standards based educational system and teachers (or even schools) can be judged objectively relative to this threshold to determine whether, on average students are demonstrating a year's worth of growth on state standardized tests. This adds an additional layer of meaning to CR-VA scores that can increase their signaling power. 
Other student characteristics are generally present in econometric models, which explicitly control for student characteristics. These values must also be fixed to specify the barely-proficient standardizing student. The easiest solution is to set the demographic characteristics to their average values in the population, taking advantage of the fact that the standardizing student is not a real person, but a useful construct. In this case, the CR-VA scores are the predicted test scores the average barely proficient student would have received in teachers' classrooms. This choice, though, is mostly arbitrary and the student demographic values could instead be selected to make the lowest (or highest) possible predicted score. In this case, the CR-VA scores are the estimated test scores for the most (least) difficult to teach barely proficient student. The specific interpretation of the CR-VA scores are altered somewhat by the choice of how to fix the standardizing student's demographic characteristics, but there is no substantive difference in scores based on how student characteristics are selected.

\section{Constructing the VA scores}

We turn now to a demonstration of how CR-VA scores are constructed. While these scores can be estimated by any VA model, we demonstrate this approach for an econometric style model commonly found in research studies (Harris \& McCaffrey, 2010). The only requirement for the CR-VA model is that a single student's score can be predicted. This is also possible for EVAAS and the growth percentile models, the two other common VA models (Collins \& Amrein-Beardsley, 2014). Consider the VA model below

$$
g\left[A_{j k t}\right]=f_{k}\left(g\left[A_{j k(t-1)}\right], X_{j k}, v_{k}\right)=g\left[A_{j k(t-1)}\right] \cdot \gamma+v_{k}+X_{j k} \cdot \beta+\varepsilon_{j k t}
$$

where $A_{j k t}$ is the test score ${ }^{3}$ of student $\mathrm{j}$ in teacher k's class at time t, $A_{j k(t-1)}$ is the student's prior year's test score (ideally test scores from multiple prior years and multiple subjects or polynomial functions of such scores are included). $g$ is an invertible, monotonic transformation function to adjust the scale of the test scores. The $g$ is included simply to 
highlight that models can be run on a convenient scale and then transformed using the inverse $\left(g^{-1}\right)$ onto a scale familiar to teachers since scales are generally monotonic transformations of other scales. $v_{k}$ is a vector of teacher fixed-effects, $X_{j k}$ is a vector of student characteristics, such as free-reduced priced lunch, special education status, English language status, etc., and $\varepsilon_{j k t}$ is a residual term.

Typically, the teacher fixed-effects (i.e. $\hat{v}_{k}$ ) are extracted as the teacher VA score estimates from the above equation. Instead, we can predict, using the same model, the test score for the barely-proficient standardizing student (i.e. $A_{j(t-1)}=c_{\text {prof }} \stackrel{\text { def }}{=}$ proficiency cutscore) as a teacher's CR-VA score. This gives a teacher CR-VA score estimate of $f_{a}\left(a \mid A_{j(t-1)}=c_{p r o f}, \hat{v_{a}}, X_{\bar{J}}, \hat{\gamma}, \hat{\beta}\right)$ for teacher a, where $X_{\bar{J}}=(1 / J) \sum_{j} X_{j}$ is the sample average values of $X_{j}$. Thus, the new CR-VA scores simply scale the traditional VA scores, moving from $\hat{v}_{a}$ to $\hat{v}_{a}+\left(g\left[c_{\text {prof }}\right] * \hat{\gamma}+X_{\bar{J}} \cdot \hat{\beta}\right)$. Hence, the correlation with the traditional VA scores should be 1 (before transforming with $g^{-1}$ ), the ranking of teachers will remain the same, and the other characteristics of the score (e.g., unbiasedness) should remain. Thus, while we gain the important benefits of more interpretable scores, non-normative scores, and criterion-referenced scores, we retain the known properties of VA scores (good and bad). In fact, the same normative interpretations of CR-VA scores is still possible and conclusions will remain the same when making judgements from this normative approach ${ }^{4}$. We also retain the known challenges of VA scores, including summer learning decay, teachers' residual effects, teachers'/students' conflated/peer effects.

\section{Challenges of CR-VA Scores}

There are a few challenges that are introduced in CR-VA scores that merit discussion. Namely the use of the student scale score metric makes scores grade-dependent and 
dependent on a specific test and test scaling. This precludes vertically equating across grades. In some sense, though, it is difficult to determine if this is a negative. VA scores, as currently estimated, typically are centered within grade, making the assumption that teacher quality does not vary across grades and concerns have been raised about how VA scores function across grades (Harris \& Anderson, 2017). Observation instruments, on the other hand, suggest that teaching quality decreases at higher grades (e.g., Grossman, Cohen, \& Brown, 2014). The net result of this lack of vertical equating is that teachers will have separate VA scores for each grade they have taught. Similarly, shifts in the test and/or scale score used will result in CR-VA scores that cannot be aligned, unless the strong assumption that there is a true linear transformation between scales is made. Equating scores across time and within-grade and within-test, on the other hand, occurs naturally and automatically through the use of the standardizing student (i.e. the standardizing student ensures scores are on same scale across years and scores can thus be averaged). Because VA scores are typically averaged across up to three years and since teachers change grades and states change test with great regularity, this means a break with current practice if CR-VA scores are used. However, this break can be seen as relaxing implicit assumptions that teacher quality is equal across grades and remains comparable across shifts in tests. On the other hand, the reduced averaging over time means that attention to uncertainty in scores and helping practitioners appropriately understand uncertainty is needed.

\section{Summary}

In this paper, we argued that, to be more effective signals of teacher quality that practitioners can use in combination with other data sources, VA scores should be placed on a scale familiar to practitioners, such that practitioners are able to reason about the meaning of scores and differences between scores. The student scale score metric seems an appropriate choice. We further argued for placing benchmark assessment scores on this 
metric to build practitioners facility in interpreting the instructional meaning of differences of scores on this metric.

In the process of mapping scores to the student scale score metric, we showed how the predicted performance of a standardizing student can be used as a VA score that is not normative, but rather both allows VA scores to be interpreted without comparison to other teachers and provides a clear standard of proficiency for teachers. In this way, the criterionreferenced VA (CR-VA) scores introduced here improve upon two major problems of VA scores, the difficulty in understanding scores and their normative nature. Last, we hypothesized that these new scores may be more trusted by teachers because they appear to fluctuate less across years.

Beyond these benefits, the proposed CR-VA scores in this paper are simply a rescaling of current scores, so important known properties of VA scores will be preserved. Importantly, this means that the many challenges inherent in VA scores (e.g., summer learning decay, properly accounting for teacher and non-teacher effects, peer effects, comparing teachers in disparate contexts) are still present. CR-VA scores help address only the three identified problems without affecting other challenges of VA scores.

\section{Practitioner Benefit}

The goal of this paper was to address problems with VA scores that will make scores more useful to practitioners. Given concerns about VA scores promoting competitiveness among teachers (Cohen \& Goldhaber, 2016; Collins, 2014; Kappler Hewitt, 2015; Johnson, 2015), the fact that CR-VA scores are a model-based estimate of non-normative scores should inherently have some benefit for teachers. Combined with data-based decisionmaking procedures, we think the CR-VA scores will be more interpretable and valued by teachers. CR-VA scores will provide a more objective and clear signal of whether a teacher's students are showing a year's worth of test score growth, increasing the signaling capacity of 
CR-VA scores over VA scores. However, we are somewhat skeptical that a single score, representing a full year of instruction will have any large or meaningful effect on teachers directly, especially given the current distrust of VA scores and the punitive way they are often used. The culture of a school will have the largest impact on how CR-VA scores are received and used by teachers and CR-VA scores are unlikely to impact school culture (Bryk \& Schneider, 2002).

The main benefit of CR-VA scores is, instead, for administrators. As we argued above, not only are criterion-referenced VA scores more interpretable, but differences in scores are more meaningful too (especially when embedded in data-based decision-making systems). This enables principals to more efficiently use VA scores as a decision tool, in combination with other pieces of evidence. Tradeoffs between various teacher characteristics can be more efficiently considered when making employment decisions about teachers. Principals will also receive a more clear, criterion-based signal of teacher quality (which could also be provided at the school level or for specific grades and/or subjects), which will provide a better view of their school's quality.

Of course, the benefit that comes with this more clear signal is dependent on how teachers and administrators react to the signal. This suggests a possible venue for future research: the need to empirically explore the impact of using CR-VA scores on administrators' data-based decision-making practices. Because CR-VA scores are created using the same statistical models on the same data, creating CR-VA scores for a small subset of schools in order to empirically test the effect of these scores should be straightforward.

\section{Policy Benefit}

There is a desire to understand whether American schools are effective in educating students, as evidenced by test scores (see Braun, 2015). While status measures often play a role in this discussion (e.g., NAEP scores and international comparisons), the question of 
whether schools are effective is better answered by growth measures (AERA, 2015). VA scores do not currently allow policy makers to understand whether schools or teachers are generating a year's worth of test score growth because they can only rank teachers without determining whether teachers are providing adequate student learning. CR-VA scores, on the other hand, provide the opportunity to conclude whether teachers and/or schools are effective (at raising test scores) because they are linked to a clear proficiency criterion, namely the criterion inherent within the current standards based accountability system as instantiated through testing and proficiency cut-scores. The percentage of teachers who meet the criterion (i.e. for whom the barely-proficient student reaches proficiency) can be calculated to determine whether the schools and teachers are effective. Further, because the basis of the criterion is the underlying student test scores, which are tied to student performance standards, the criterion seems particularly appropriate to determine whether schools are effective within the context of a standards-based system.

\section{Plan of Study}

Here, we set forth a plan for studying the impact and consequences of CR-VA scores. First, it is important to reiterate that there are two interpretations of CR-VA scores. CR-VA scores can be interpreted in the standard normative fashion, where teacher scores are compared to each other. This interpretation is most appropriate when deciding between employment decisions across teachers, where the key question becomes which teacher is better for a given assignment. In this mode, for scores within a single year, there is little to verify from a statistical standpoint, since scores are a simple rescaling of existing scores, inheriting the statistical properties from typical VA scores (e.g., reliability, validity, bias, or other issues). CR-VA scores, though, are grade-specific and research will have to explore how much this hampers aggregated scores across years to reduce standard errors. Research should also explore whether teachers are ranked similarly in different grades. However, the 
main topic of study would be how teachers and principals interpret and understand scores. This could be studied through surveying principals, where principals make fake personnel decisions (e.g., hiring, granting tenure, or dismissal) based on resumès crafted with either standard or CR-VA scores and the outcome is whether principals tend to make "better" personnel decisions when provided with CR-VA scores. Of course, determining what constitutes a "better" decision is challenging and such studies will depend heavily on the way different scores are presented. Further, as we have argued scores should appear to change less across time (in terms of raw numerical shifts), so it will be important to explore how teachers make sense of their scores across time, whether they perceive variation as more modest, and whether trust is scores is better developed. This could be explored through presenting teachers with mock reports of their and see how they understand those scores.

Of course, as we have argued throughout, the benefit of CR-VA scores is maximized when combined with data-based decision-making efforts that build understanding of differences of scores on the student scale score metric. Because of this, the best test of the usefulness of CR-VA scores would be in the context of a full district pilot that involves aligning benchmark assessments in addition to CR-VA scores.

When CR-VA scores are interpreted in a criterion-referenced manner, as would be appropriate when seeking to understand whether teachers, schools, or school systems are effective, more basic statistical work is needed. Namely, scores with a criterion-referenced interpretation have greater error, because additional sources of error such as the regression residual are now relevant ${ }^{5}$. Research would have to examine how much additional unreliability this adds to CR-VA scores. Additionally, policy research examining the distribution of teacher and school quality across contexts would be important here. For example, the basic question of what percentage of the teachers is providing a years' worth of 
growth on test scores (and how does this vary across the characteristics of students served) is important.

\section{Conclusion}

In this paper, we have put forth a method to address some of the concerns practitioners have raised regarding VA scores. The end result, which we call criterion-referenced VA scores, amounts to a simple rescaling of scores, but has the promise to make scores more interpretable and meaningful while removing the normative nature of scores that may impact teacher cooperation. While much work remains to test whether the new CR-VA scores will actually remove negative impact VA measures have on teacher cooperation and increase their ability to signal teacher quality, the theory developed in this paper has the potential to make VA scores a more meaningful policy tool. 


\section{Bibliography}

American Educational Research Association (AERA). (2015). AERA statement on use of value-added models (VAM) for the evaluation of educators and educator preparation programs. Educational Researcher, 44(8), 448-452. https://doi.org/10.3102/0013189X15618385

American Statistical Association. (2014). ASA Statement on Using Value-Added Models for Educational Assessment. Retrieved from https://www.amstat.org/asa/files/pdfs/POLASAVAM-Statement.pdf

Beaver, J. K., \& Weinbaum, E. H. (2015). State Test Data and School Improvement Efforts. Educational Policy, 29(3), 478-503.

Betebenner, D. W. (2009). Norm- and Criterion-Referenced Student Growth. Educational Measurement: Issues and Practice, 28(4), 42-51. https://doi.org/10.1111/j.1745$\underline{3992.2009 .00161 . x}$

Braun, H. (2015). The Value in Value Added Depends on the Ecology. Educational Researcher, 44(2), 127-131. https://doi.org/10.3102/0013189X15576341

Brennan, R. L. (2001). Generalizability Theory. New York, NY: Springer New York. Retrieved from http://link.springer.com/10.1007/978-1-4757-3456-0

Cohen, J. J., \& Goldhaber, D. (2016). Building a More Complete Understanding of Teacher Evaluation Using Classroom Observations. Educational Researcher, 45(6), 378-387. https://doi.org/10.3102/0013189X16659442

Farrell, C. C., \& Marsh, J. A. (2016). Contributing conditions: A qualitative comparative analysis of teachers' instructional responses to data. Teaching and Teacher Education, 60, 398-412. 
Goe, L., \& Croft, A. (2009). Methods of Evaluating Teacher Effectiveness. Research-toPractice Brief. National Comprehensive Center for Teacher Quality. Retrieved from http://eric.ed.gov/?id=ED543666

Goldring, E., Grissom, J. A., Rubin, M., Neumerski, C. M., Cannata, M., Drake, T., \& Schuermann, P. (2015). Make Room Value Added Principals’ Human Capital Decisions and the Emergence of Teacher Observation Data. Educational Researcher, 44(2), 96-104.

Grossman, P., Cohen, J. J., \& Brown, L. (2014). Understanding Instructional Quality in English Language Arts: Variations in PLATO Scores by Content and Context. In Designing teacher evaluation systems: New guidance from the measures of effecting project (pp. 303-331). San Francisco, CA: Jossey-Bass.

Guarino, C., Reckase, M., Stacy, B., \& Wooldridge, J. (2015). A Comparison of Student Growth Percentile and Value-Added Models of Teacher Performance. Statistics and Public Policy, 2(1), 1-11. https://doi.org/10.1080/2330443X.2015.1034820

Harris, D. N., \& Anderson, A. (n.d.). Does Value-Added Work Better in Elementary Than in Secondary Grades? 13.

Ingram, D., Louis, K. S., \& Schroeder, R. G. (2004). Accountability policies and teacher decision making: Barriers to the use of data to improve practice. Teachers College Record, 106(6), 1258-1287.

Kappler Hewitt, K. (2015). Educator Evaluation Policy that Incorporates EVAAS ValueAdded Measures: Undermined Intentions and Exacerbated Inequities. Education Policy Analysis Archives, 23(0), 76. https://doi.org/10.14507/epaa.v23.1968

Newton, X. A., Darling-Hammond, L., Haertel, E., \& Thomas, E. (2010). Value-Added Modeling of Teacher Effectiveness: An Exploration of Stability across Models and 
Contexts. Education Policy Analysis Archives, 18. Retrieved from http://www.redalyc.org/resumen.oa?id=275019712023

Reardon, S. F., \& Raudenbush, S. W. (2009). Assumptions of Value-Added Models for Estimating School Effects. Education Finance and Policy, 4(4), 492-519. https://doi.org/10.1162/edfp.2009.4.4.492

Rubin, D. B., Stuart, E. A., \& Zanutto, E. L. (2004). A Potential Outcomes View of ValueAdded Assessment in Education. Journal of Educational and Behavioral Statistics, 29(1), 103-116.

Schacter, J., \& Thum, Y. M. (2004). Paying for high- and low-quality teaching. Economics of Education Review, 23(4), 411-430. https://doi.org/10.1016/j.econedurev.2003.08.002

Wachen, J., Harrison, C., \& Cohen-Vogel, L. (2018). Data Use as Instructional Reform: Exploring Educators' Reports of Classroom Practice. Leadership and Policy in Schools, 17(2), 296-325. 


\section{Notes}

1. Another potential use of VA scores is to provide teachers information to improve instruction, but there is general skepticism that a single score could provide detailed guidance for how to improve (Cohen \& Goldhaber, 2016; Goldring, et al., 2015) so we focus on the use of VA scores for employment decisions.

2. We would, of course, prefer that benchmarks be instructionally sensitive, relevant, and useful and that data-based decisions utilized information that broke down student performance across learning objectives.

3. We tacitly assume the test scores (i.e. the $A_{j k(t-1)}$ terms) are in the scale score metric, rather than the z-scored or equi-percentile metrics frequently used. This assumption is mainly for expository clarity as any monotonic, invertible function can be applied to the test score terms in the model (i.e. $\left.g\left(A_{j k(t-1)}\right)\right)$ if the predicted scores get transformed back to the appropriate scale (i.e. $g^{-1}\left[\hat{f}_{k}\left(c_{\text {prof }}, X_{j k}, \hat{v_{k}}\right)\right]$ ). That said, using the scale scores metric likely makes the CR-VA scores slightly less robust to the traditional interval level assumption made by all VA models (Reardon \& Raudenbush, 2009).

4. The careful reader will notice that this approach has some implications for the standard error of the teacher score estimate. There are two types of standard errors to consider here, analogous to relative and absolute reliabilities from generalizability theory (Brennan, 2001). When comparing teachers' scores to each other, any error inherent to the VA model is constant across teachers. Thus, the standard errors of the teacher fixedeffects (i.e. $s e\left(\hat{v}_{k}\right)$ ) should be used, giving the same standard errors as traditional VA scores. However, when comparing a teacher's score to a fixed score value. For example, when determining whether students of a given teacher had a year's worth of growth on test scores. The uncertainty in the model parameters (i.e. $s e(\hat{\gamma})$ and $s e(\hat{\beta}))$ as well as 
the residual model error (i.e. $\left.\sqrt{\operatorname{var}\left(\varepsilon_{j k t}\right)}\right)$ contribute to the uncertainty in CR-VA scores, giving larger standard errors. It is not clear how much this might inflate standard errors. But again, this increase in standard errors comes only when trying to compare teachers to a fixed value that the barely proficient student should reach, not when using VA scores as they are currently used. Care should be taken in estimating these standard errors as software usually does not include uncertainty in model parameter estimates when creating standard errors for predicted scores.

5. Specifically, the additional error arises from $\operatorname{se}(\hat{\gamma}), \operatorname{se}(\hat{\beta})$, and $\operatorname{se}\left(\widehat{\varepsilon_{\jmath k} t}\right)$ 